\title{
An Intelligent Multimedia Data Encryption and Compression and Secure Data Transmission of Public Cloud
}

\author{
Sheik Saidhbi \\ Assistant Professor, Department of Information Systems, Faculty of Informatics, University of Gondar, Ethiopia \\ E-Mail: sfajju.syed@gmail.com
}

\begin{abstract}
Data compression is a method of reducing the size of the data file so that the file should take less disk space for storage. Compression of a file depends upon encoding of file. In lossless data compression algorithm there is no data loss while compressing a file, therefore confidential data can be reproduce if it is compressed using lossless data compression. Compression reduces the redundancy and if a compressed file is encrypted it is having a better security and faster transfer rate across the network than encrypting and transferring uncompressed file. Most of the computer applications related to health are not secure and these applications exchange lot of confidential health data having different file formats like HL7, DICOM images and other audio, image, textual and video data formats etc. These types of confidential data need to be transmitted securely and stored efficiently. Therefore this paper proposes a learning compression- encryption model for identifying the files that should be compressed before encrypting and the files that should be encrypted without compressing them.

Keywords: Encryption, Compression, Data Security, ECB
\end{abstract}

\section{INTRODUCTION}

Data compression is a method of reducing the size of the data file so that the file should take less disk space for storage [11]. The file that contains redundancy gets reduced by compression. In lossy data compression algorithms there is loss of original data while performing compression [20]. In computer science and Information technology, a data encoding method in which the data is compressed by losing some amount of data is lossy compression.

\section{A. Lossless Data Compression Algorithms}

In case of lossless data compression algorithm there is no data loss while compressing a file, it guarantees to reproduce the exactly same data as input. If data loss is not desirable the lossless data compression algorithms should be used. Some of the peculiar examples include executable text documents, programs and source codes etc. Some of the image file formats also uses lossless compression.

Huffman Code-It assign more bits to symbols appear less and fewer bits to the symbols that occur more frequently. Every Huffman code having the same average code length. It optimizes the single byte at time.

1. Deflate- It combines the LZ77 and Huffman code for compression in which LZ77 optimizes sequence of bytes whereas Huffman works on single byte.

2. LZ4-It lossless data compression algorithm that is primarily focused on compression and decompression speed. The algorithm gives a slightly worse compression ratio than others.

3. LZF- It is a fast compression algorithm that takes very little working memory and code space.

4. Zip- Zip is a lossless compression Algorithm. Zip compress as well as archive the file. Content of the .Zip file can be a single file of group of files enclosed in a folder.

\section{B. Cryptographic Algorithms}

Symmetric key cryptographic ciphers come in two types, stream and block ciphers. Stream ciphers works on bits stream or bytes stream. Stream ciphers are used for securing data of terminal and wireless applications. Block ciphers performs encryption or decryption on fixed size block of data. In network applications block ciphers are used for transmission of files of huge sizes which require high security. Deciphering cipher text without knowing the key is called cryptanalysis. Cryptanalysis of block ciphers is difficult compared to stream ciphers [9]. Hence in most of the applications, block ciphers are used for providing better security than stream ciphers.

Block ciphers come in various block modes. Block mode for cipher algorithm determines how cipher text blocks are created by encryption from plaintext blocks and vice versa. $\mathrm{ECB}, \mathrm{CBC}, \mathrm{CFB}, \mathrm{OFB}, \mathrm{PCBC}$ and CTR etc are commonly used block modes [9]. ECB has poor security properties since encryption of a block with a fixed size always yields the same result; hence susceptible to dictionary attacks, replay attacks etc. In case of CBC first plaintext block is XORed with IV and remaining all plaintext blocks are XORed with previous cipher text blocks; while in case of PCBC operation on first plaintext block is similar to CBC but remaining all plaintext blocks are XORed with previous plaintext as well as previous cipher text block [9] [12].

In paper [15], the author has simulated different symmetric key cryptographic algorithms like AES, DES, 3-DES and Blowfish. The simulation was done on 0.5 to

20MB data blocks. The simulation results show that the Blowfish yields better results than other symmetric key 
cryptographic algorithms when it comes to processing power. AES yield poor results as it requires high processing power. Initially all the simulations were taken in ECB mode and it was observed that Blowfish takes comparatively less processing time than others. AES takes relatively higher time when the block size is high. It was also concluded that 3-DES will always take more time as compared to 3-DES as it involves 3 phases of encryption. Another simulation was done on all the symmetric key algorithms in CBC mode. It was concluded that $\mathrm{CBC}$ took more time for performing encryption than ECB mode.

\section{DESIGN OF MODEL OF COMPRESSION ALGORITHM FOR DATA TYPE ANALYSIS}

Every compression algorithm has different level of compression for different files. For example, there are two compression algorithms A and B. Suppose compression algorithm $\mathrm{A}$ has compression ratio of $30 \%$ for $\mathrm{X}$ file. It is not necessary that compression algorithm $B$ will have same compression Ratio for X file. Compression Algorithm B can give more, less or no compression at all. This sub section provides information on calculating the compression ratio for different compression algorithms. The following algorithm describes the process for calculating compression ratio for a file.

\section{Procedure Name: Compress}

Input Parameters: input File: Name of input file for compression

\section{Start Procedure}

Array $=$ Read all the compression algorithm in Array

Read input file for compression

Determine the data type from file extension

Find uncompressed size of file for $i=1$ to Array Length

Compress file by Compression Algo[i] Calculate compressed file size;

Calculate compression Ratio by eq. 1

End for

Write the compression ratio to database

\section{End Procedure}

All cipher algorithms are implemented using sun provider except skipjack, which is implemented using Bouncy Castel provider. Fo llowing is the piece of code used for analysis:

$/ / B C=$ Bouncy Castel Provider cipher = Cipher.getInstance(algorithmName, “BC”);

// for encryption

operation $=$ Cipher.ENCRYPT_MODE;

//Initializing cipher cipher.init(operation, secretKey);

//Performing Encryption

encryptOutLength $=$ cipher.update $($ inputBytes, 0 , bufferSize, outputBytes);

encryptOutLength $=$ cipher.doFinal(inputBytes, 0 , inLength);

// for decryption

operation $=$ Cipher.DECRYPT_MODE;

//Initializing cipher cipher.init(operation, secretKey); //Performing Decryption

decryptOutLength $=$ cipher.update $($ inputBytes, 0 , bufferSize, outputBytes);

decryptOutLength $=$ cipher $\cdot$ doFinal $($ inputBytes, 0 , inLength);

From the related works, it is realized that none of the work did a very detailed analysis of the performance of various symmetric algorithms, on various parameters of different type of files. In order to select the most suitable cryptographic algorithm for encryption, following test cases are considered to analyze the time taken for encryption by various cryptographic algorithms.

A data file format represents the standard for encoding the information to be stored in computer file. This case study is taken to check whether the encryption has dependency on type of data or not. Different data type files like audio, image, textual, video and health data file format like DICOM of nearly 50MB, and 100MB in size are chosen and encryption time of different cipher algorithms is calculated for these data.

\section{A. Data Files of Same Type with Different Sizes}

This case study is taken to ensure once again the observations obtained from case study 1, that encryption time depends on number of bytes in the file. In this study is, different files of same types but different sizes are given for encryption and estimated the encryption time. For all executions, key size and block mode are kept at bare minimal parameters.

Table I gives the details about the files used for all executions and Figure 3, 4 and 5 show the execution results. 
TABLE I EXECUTION PARAMETERS For FILES OF DifFERENT Sizes

\begin{tabular}{|c|c|c|}
\hline File Type & $\begin{array}{c}\text { Varying Parameters } \\
\text { (Data Size) }\end{array}$ & $\begin{array}{c}\text { Constant } \\
\text { Parameters }\end{array}$ \\
\hline AIFF & $\begin{array}{c}10.7 \mathrm{MB}, 50 \mathrm{MB}, \\
100 \mathrm{MB}\end{array}$ & \multirow{3}{*}{$\begin{array}{c}\text { Data Type, Key } \\
\text { size, Block Mode }\end{array}$} \\
\hline AVI & $\begin{array}{c}50 \mathrm{MB}, 100 \mathrm{MB}, \\
482 \mathrm{MB} \\
\end{array}$ & \\
\hline DICOM & $\begin{array}{c}14.9 \mathrm{MB}, 50.3 \mathrm{MB}, \\
115 \mathrm{MB}, \\
151 \mathrm{MB}\end{array}$ & \\
\hline
\end{tabular}

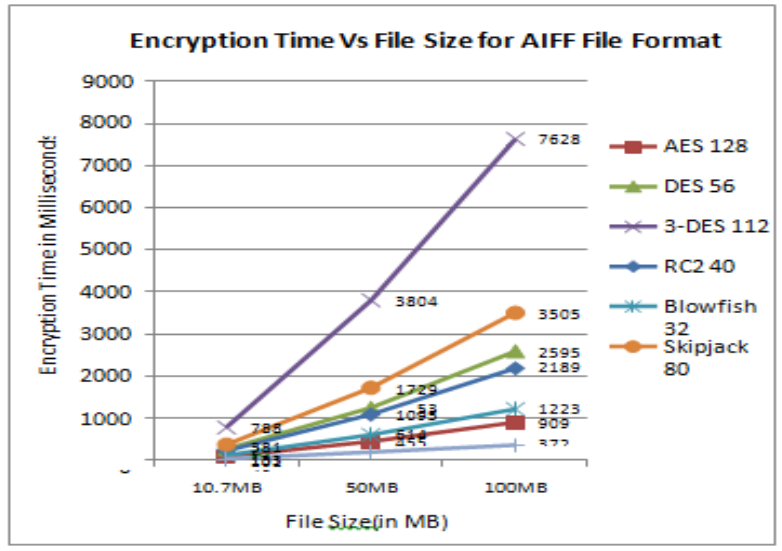

Fig. 1 File size Vs Encryption time for AIFF file of different sizes

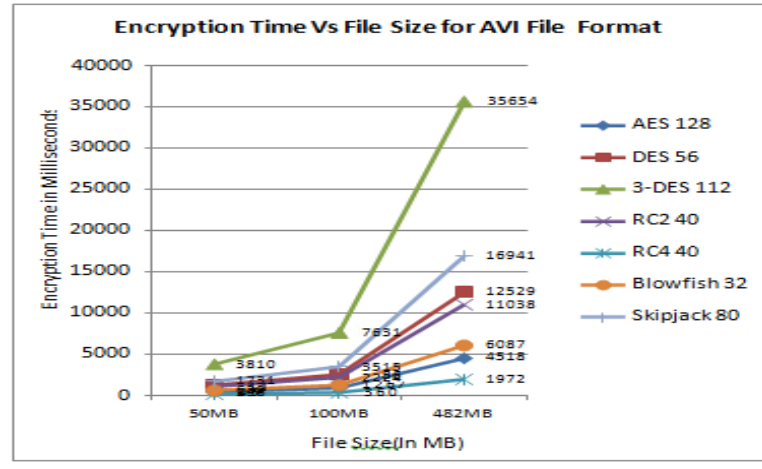

Fig. 2 File size Vs Encryption time for AVI file of different sizes

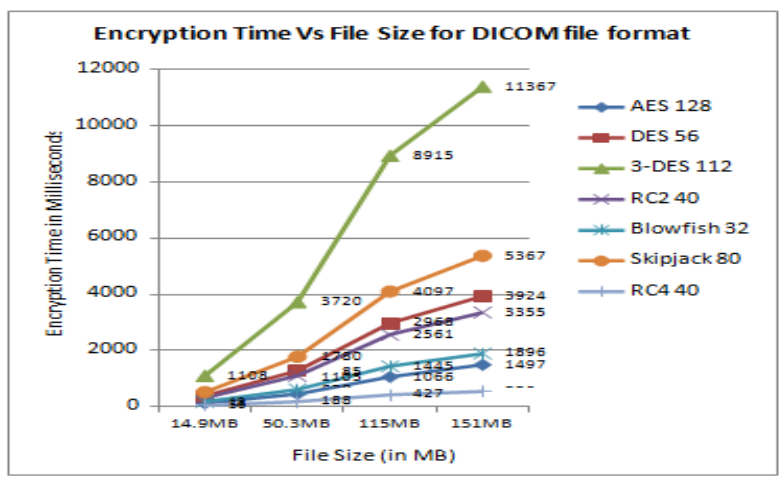

Fig. 3 File size Vs Encryption time for DICOM file of different sizes

TABLE II ENCRYPTION RATE For FiLES OF DIFFERENT SizES

\begin{tabular}{|c|c|c|c|c|c|c|c|c|}
\hline \multirow{2}{*}{$\begin{array}{l}\text { File } \\
\text { Type }\end{array}$} & \multirow{2}{*}{$\begin{array}{c}\text { Size } \\
\text { (In MB) }\end{array}$} & \multicolumn{7}{|c|}{ Encryption Rate In (MB/sec) } \\
\hline & & $\begin{array}{c}\text { AES } \\
128 \\
\end{array}$ & $\begin{array}{c}\text { DES } \\
56 \\
\end{array}$ & $\begin{array}{c}\text { 3-DES } \\
112\end{array}$ & $\begin{array}{c}\mathrm{RC2} \\
40 \\
\end{array}$ & $\begin{array}{c}\text { Blowfish } \\
32 \\
\end{array}$ & $\begin{array}{c}\text { Skipjack } \\
80 \\
\end{array}$ & $\begin{array}{c}\mathrm{RC4} \\
40 \\
\end{array}$ \\
\hline \multirow{3}{*}{ AIFF } & 10.7 & 109.18 & 39.43 & 13.61 & 45.06 & 80.63 & 28.14 & 268.12 \\
\hline & 50 & 109.95 & 39.92 & 13.15 & 45.68 & 81.47 & 28.93 & 270.66 \\
\hline & 100 & 110.07 & 38.55 & 13.11 & 45.71 & 81.81 & 28.54 & 268.96 \\
\hline \multirow{3}{*}{ AVI } & 50 & 107.83 & 39.49 & 13.14 & 45.04 & 79.62 & 28.93 & 265.52 \\
\hline & 100 & 109.3 & 38.8 & 13.14 & 45.11 & 79.19 & 28.54 & 270.72 \\
\hline & 482 & 108.74 & 38.49 & 13.52 & 45.09 & 79.22 & 28.46 & 265.55 \\
\hline \multirow{4}{*}{ DICOM } & 14.9 & 107.65 & 38.93 & 13.45 & 45.46 & 80.61 & 28.19 & 266.31 \\
\hline & 50.3 & 108.48 & 39.17 & 13.53 & 45.63 & 81.19 & 28.28 & 267.76 \\
\hline & 115 & 108.69 & 39.03 & 12.99 & 45.24 & 80.18 & 28.28 & 271.34 \\
\hline & 151 & 108.21 & 38.61 & 13.32 & 45.15 & 79.91 & 28.23 & 270.47 \\
\hline \multicolumn{2}{|c|}{ Average } & 108.8 & 39.04 & 13.3 & 45.32 & 80.383 & 28.452 & 268.5 \\
\hline
\end{tabular}

\section{B. Files with Different Densities of Data}

Encryption rate is evaluated for different files, a sparse AIFF file of $69 \mathrm{MB}$ and a dense AIFF file of 58.5MB. For a cipher algorithm, key size and block mode are kept at bare minimal parameters. The results of execution are shown in Table III.

\section{Cipher Algorithms with Different Block Modes}

Security of cipher algorithm also varies according to block cipher modes. Different block cipher modes are used for different applications. For example PCBC is used in
WASTE and Kerberos v4. Security levels may differ according to type of application and can be classified as:

This study is to check the encryption time variation with respect to block cipher modes. All block cipher algorithms have been executed for different block modes with PKCS\#5 padding scheme on 50.5MB DICOM file. The key size for particular block cipher algorithm is kept at the bare minimal value. The various block modes mentioned in Table II are used for evaluation. The Figure 4 shows the block cipher variation for AES 128 and Figure 5 shows the result of execution for all encryption algorithms. 
TABLe III ENCRyption RATE For SPARSE AND DENSE Data File

\begin{tabular}{|l|c|c|c|c|}
\hline \multirow{2}{*}{$\begin{array}{c}\text { Algorithm } \\
\text { Name }\end{array}$} & \multicolumn{2}{|c|}{$\begin{array}{c}\text { Sparse } \\
\text { Dense } \\
\text { (72000118 Bytes) AIFF file }\end{array}$} & $\begin{array}{c}\text { Encrypt } \\
\text { (61392454 Bytes) AIFF file }\end{array}$ \\
\cline { 2 - 5 } & $\begin{array}{c}\text { Encryption } \\
\text { Rate(MB/s) }\end{array}$ & $\begin{array}{c}\text { Encrypt } \\
\text { Time(ms) }\end{array}$ & $\begin{array}{c}\text { Encryption } \\
\text { Rate(MB/s) }\end{array}$ \\
\hline AES 128 & 634 & 108.28 & 540 & 108.40 \\
\hline DES 56 & 1801 & 38.11 & 1537 & 38.08 \\
\hline 3-DES 112 & 5076 & 13.52 & 4365 & 13.41 \\
\hline RC2 128 & 1520 & 45.16 & 1285 & 45.55 \\
\hline Blowfish 128 & 854 & 80.38 & 723 & 80.96 \\
\hline Skipjack 128 & 2386 & 28.77 & 2042 & 28.66 \\
\hline RC4 128 & 253 & 271.35 & 216 & 271.01 \\
\hline
\end{tabular}

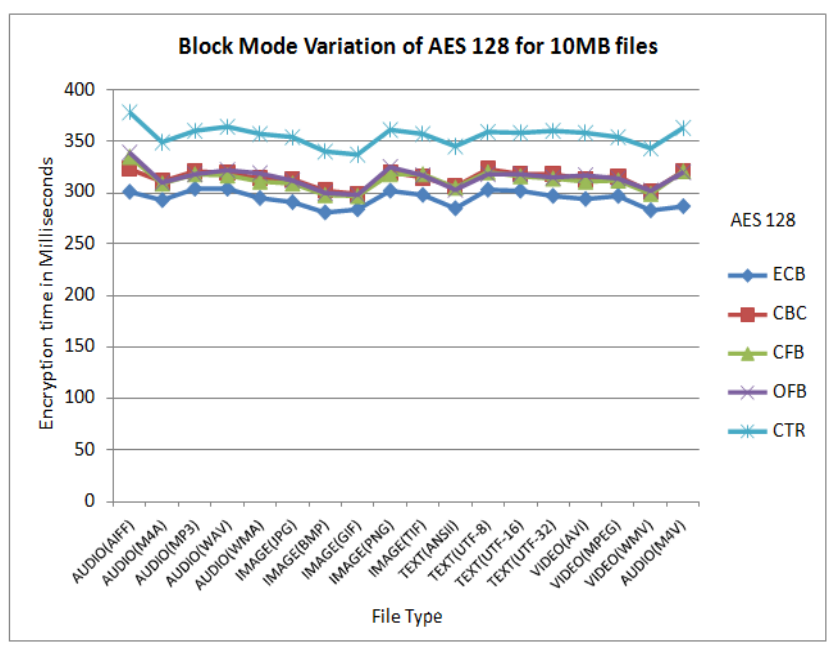

Fig. 4 Block Mode Variation of AES 128 for 10MB files

\section{CONCLUSION}

After analysis of all parameters, AES was found to be most suitable encryption algorithm having encryption rate of $108 \mathrm{MB} / \mathrm{sec}$ in ECB mode. AES was used in the proposed compression-encryption model. A compression-encryption model was proposed for identifying the files that should be compressed before encrypting and the files that should be encrypted without compressing them. A formula was derived imperially to determine best suitable compression algorithm that should be used for compressing the file according to data type and data size to reduce the overhead of time for compression and to increase the efficiency and security to data that is being transferred.

\section{REFERENCES}

[1] A. L. Jeeva, Dr. V. Palanisamy and K. Kanagaram, "Comparative Analysis of Performance Efficiency and Security Measures of Some Encryption Algorithms", International Journal of Engineering Research and Applications (IJERA), Vol. 2, No. 3, pp.3033-3037, May-Jun 2012.
[2] S. Soni, H. Agrawal and M. Sharma, "Analysis and comparison between AES and DES Cryptographic Algorithm", International Journal of Engineering and Innovative Technology, Vol. 2, No. 6, pp. 362-365, Dec. 2012.

[3] Nidhi Singhal and J. P. S. Raina, "Comparative Analysis of AES and RC4 Algorithms for Better Utilization", International Journal of Computer Trends and Technology, Vol. 2, No. 6, pp. 177-181, JulyAug 2011.

[4] Jawahar Thakur and Nagesh Kumar, "DES, AES and Blowfish: Symmetric Key Cryptography Algorithms Simulation Based Performance Analysis", International Journal of Emerging Technology and Advanced Engineering, Vol. 1, No. 2, pp. 6-12, Dec. 2011.

[5] Allam Mousa and Ahmad Hamad, "Evaluation of the RC4 Algorithm for Data Encryption", International Journal of Computer Science \& Applications, Vol. 3, No. 2, pp. 44-56, June 2006.

[6] Aamer Nadeem, M. Younus Javed, "A Performance Comparison of Data Encryption Algorithms", First International Conference on IEEE Information and Communication Technologies (ICICT), pp. 8489, 27-28 Aug. 2005.

[7] Kofahi, N.A, Turki Al-Somani, Khalid Al-Zamil, "Performance evaluation of three Encryption/Decryption Algorithms", IEEE 46th Midwest Symposium on Circuits and Systems, pp. 790-793, 30-30 Dec. 2003.

[8] Jonathan Knudsen, Java Cryptography, 2nd Edition, O’Reilly, 2011.

[9] William Stallings, Cryptography and Network Security, 5th Edition, Pearson, 2012.

[10] O.S. Pianykh, Digital Imaging and Communications in Medicine (DICOM), $1^{\text {st }}$ Edition, Springer, 2008.

[11] John Miano, Compressed Image File Formats, 1st Edition, Addison Wesley Longman, Inc, 1999.

[12] Atul Kahate, Cryptography and Network Security, 2nd Edition, Tata McGraw Hill, 2012.

[13] V.K. Pachgare, Cryptography and Information Security, 1st Edition, PHI Learning PVT LTD, 2008.

[14] Dworkin, M. NIST Special Publication 800-38A. Recommendation for block cipher Modes of operation: Modes and techniques, Dec. 2001.

[15] Simar Preet Singh and Raman Maini, "Comparison of Data Encryption Algorithms", International Journal of Computer Science and Communication (IJCSC), Vol. 2, No. 1, pp. 125-127, January-June 2011.

[16] Lalit Singh and R.K. Bharti, "Comparative Performance Analysis of Cryptographic Algorithms", International Journal of Advanced Research in Computer Science and Software Engineering (IJARCSSE), Vol. 3, No. 11, pp. 563-568, November 2013. 